\title{
Simplified PRBMs of spatial compliant multi-beam modules for planar motion
}

\author{
G. Hao \\ Department of Electrical and Electronic Engineering, School of Engineering, \\ University College Cork, Cork, Ireland \\ Correspondence to: G. Hao (g.hao@ucc.ie)
}

Received: 19 February 2013 - Revised: 23 April 2013 - Accepted: 5 June 2013 - Published: 16 September 2013

\begin{abstract}
PRBMs (pseudo-rigid-body models) have been becoming important engineering technologies/methods in the field of compliant mechanisms to simplify the design and analysis through the use of the knowledge body of rigid-body mechanisms coupling with springs. This article addresses the PRBMs of spatial multi-beam modules for planar motion, which are composed of three or more symmetrical wire/slender beams parallel to each other where the planar twisting DOF (degree of freedom) is assumed to be very small for specific applications/loading conditions. Simplified PRBMs are firstly proposed through replacing each beam in spatial multi-beam module with a rigid-body link plus two identical spherical joints at its two ends. The characteristics factor, bending stiffness and twisting stiffness for the spherical joint are determined. Loaddisplacement equations are then derived for a class of spatial multi-beam modules and general spatial multibeam modules using the virtual work principle and kinematic relationships. Finally, nonlinear FEA (finite element analysis) is employed with comparisons with the PRBMs. The present PRBMs have shown the ability to predict the primary nonlinear constraint characteristics such as load-stiffening effect, cross-axis coupling in the two primary translational directions and buckling load.
\end{abstract}

\section{Introduction}

A spatial multi-beam module is a spatial compliant mechanism/joint that has compatible size in three dimensions, which transmits motion/load through the deformation of its flexible members. This article studies a class of symmetrical-beam based spatial compliant parallel modules with distributed-compliance for planar motion ("spatial multi-beam module" in brief), which are composed of three or more parallel wire/slender beams connecting the base and the motion stage. Two primary applications for this class of spatial multi-beam modules are identified as follows:

a. The spatial multi-beam module may act as an independent motion stage actuated by the non-contact electromagnetic (EM) actuators (an example desktop-size XY compliant parallel manipulator is shown in Fig. 1). This motion stage has a very simple configuration and large out-of-plane stiffness, and has no the heat effect from the EM actuator due to the non-contact actuation. Due to the fact that the output motion stage acts as the input stage as well, no lost motion exists and fewer sensors are needed.

b. A spatial multi-beam module can also be used as the basic building block of new multi-axis compliant parallel manipulators, for example as a spatial leg to enhance the out-of-plane stiffness of an XY compliant parallel manipulator (Hao and Kong, 2012a), and as a passive PPR (P: prismatic joint, $\mathrm{R}$ : revolute joint) joint of an $\mathrm{XYZ}$ compliant parallel manipulator (Hao and Kong, 2012b) (Fig. 2).

In addition, the spatial multi-beam modules composed of wire/slender beams may promote the fabrication using the carbon nanotubes (CNTs). This may lead to novel CNTbased compliant mechanisms used in the emerging nanoelectro-mechanical-systems (NEMS) (Howell et al., 2010; Culpepper et al., 2006). 


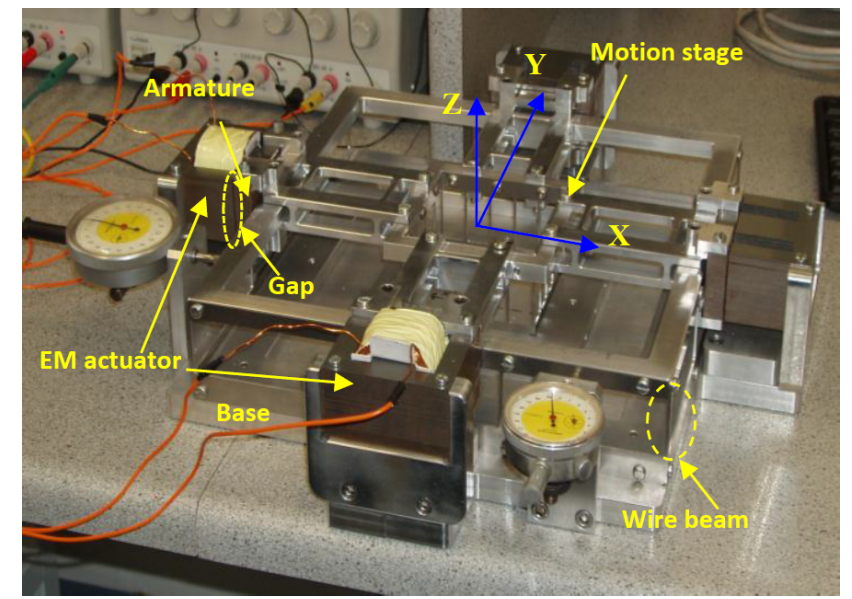

Figure 1. A desktop-size XY compliant parallel manipulator actuated by two EM actuators (where the twisting rotation about the $z$ axis is well constrained by appropriately setting up the ratio of the motion stage size to the wire beam length and making beams distribute around multiple circles).

Over the past decade, PRBMs (pseudo-rigid-body models) (Howell et al., 1996; Howell, 2001; Su, 2009; Ramirez and Lusk, 2011) have drawn plenty of attentions due to dramatically simplifying the design and analysis of compliant mechanisms using the knowledge body of rigid-body mechanisms with springs. In PRBM, the compliant beams are typically replaced with the pseudo-rigid-body link(s) coupling with one or more characteristic pivots with specified spring stiffness located at specified position(s). Most researches have been conducted for proposing PRBMs of planar-motion compliant mechanisms with planar-motion members such as the fixedfree beam, fixed-guided beam, parallelogram mechanism, cartwheel rotational joint and fixed-clamped carbon nanotubes (Howell et al., 1996, 2010; Howell, 2001; Su, 2009), which has resulted in very accurate approximation of loaddisplacement relationships. However, less work has been reported for PRBMs of spatial-motion compliant beams such as spatial-motion axisymmetric cantilever beams (Ramirez and Lusk, 2011).

This paper aims to propose a simplified PRBM of the spatial multi-beam module over intermediate range of motion (transverse bending displacement up to $10 \%$ of beam length) for the above two types of applications/loading conditions where the planar twisting DOF (degree of freedom) is constrained (very small) and only the two primary translations are left as the DOF. The present PRBM is envisaged to reflect the primary nonlinear constraint characteristics, which can detect the performance merits and shortcomings to enable the quick design synthesis.

The rest sections of this article are organized as follows. Section 2 derives the simplified PRBMs of spatial multibeam modules. In Sect. 3, FEA results are illustrated to verify the PRBM of the spatial three-beam module. Some improve- ment suggestions are discussed in Sect. 4. Finally, conclusions are drawn.

\section{Simplified PRBMs of spatial multi-beam modules}

\subsection{Simplified PRBM for a fixed-fixed beam in planar motion}

The simplified PRBM of a fixed-fixed beam with a length of $L$ in planar motion has been suggested by the previous work (Howell, 2001) where two rotational joints with each rotational spring stiffness $K_{\mathrm{b}}$ are each located the same distance from their respective end and the rigid-body link has a length of $\gamma L$. These arguments may provide a strong reference for the PRBM of the fixed-fixed beam in spatial motion in a straightforward way. The bending stiffness, $K_{\mathrm{b}}=2 \gamma K_{\theta} E I / L$ ( $K_{\theta}$, bending stiffness coefficient, $E$, Young's Modulus, and $I$, second moment of cross-section area), and the characteristic factor, $\gamma$, can be obtained from the analytical nonlinear model of a parallelogram flexure module composed of two fixed-fixed leaf beams (Awtar and Slocum, 2007) as derived below.

The virtual work principle (Howell, 2001) for the parallelogram flexure module yields

$F_{\mathrm{y}} \mathrm{d} Y_{\mathrm{s}}+P \mathrm{~d} X_{\mathrm{s}}=\frac{\partial U}{\partial Y_{\mathrm{s}}} \mathrm{d} Y_{\mathrm{s}}$

where the variable $Y_{\mathrm{s}}$ is the generalized coordinate that is the primary translational displacement of the motion stage center along the $y$ axis. $X_{\mathrm{s}}=-\left[1-\cos \left(\frac{Y_{\mathrm{s}}}{\gamma L}\right)\right] \gamma L=-\left(\frac{Y_{\mathrm{s}}}{\gamma L}\right)^{2} \gamma L / 2$, which is the parasitic translational displacement of the motion stage along the $x$ axis (see Fig. 3 for the details). $F_{\mathrm{y}}$ and $P$ are the transverse force and the axial force along the $y$ and $x$ axes, respectively, and $U$ is the total elastic energy from the deformation contribution of four rotational joints, which is equal to $4 \times 0.5 K_{\mathrm{b}}\left(\frac{Y_{\mathrm{s}}}{\gamma L}\right)^{2}$. Here, $\frac{Y_{\mathrm{s}}}{\gamma L}$ is used to denote the rotational displacement, $\theta_{z}$, of each rotational joint.

Differentiating Eq. (1) with respect to $Y_{\mathrm{s}}$ produces

$$
\begin{aligned}
& F_{\mathrm{y}}-P \mathrm{~d}\left[\left(\frac{Y_{\mathrm{s}}}{\gamma L}\right)^{2} \gamma L / 2\right] / \mathrm{d} Y_{\mathrm{s}}=\frac{\mathrm{d}\left[4 \times \frac{1}{2} K_{\mathrm{b}}\left(\frac{Y_{\mathrm{s}}}{\gamma L}\right)^{2}\right]}{\mathrm{d} Y_{\mathrm{s}}} \\
& \Rightarrow \\
& Y_{\mathrm{s}}=\frac{F_{\mathrm{y}}}{4 K_{\mathrm{b}} /(\gamma L)^{2}+P /(\gamma L)}
\end{aligned}
$$

In addition, the closed-form analytical solution for the primary translational displacement of the parallelogram flexure module is shown below (Awtar and Slocum, 2007):

$$
\begin{aligned}
Y_{\mathrm{s}} & =\frac{F_{\mathrm{y}} /\left(E I / L^{2}\right)}{2 a+P e /\left(E I / L^{2}\right)} L=\frac{F_{\mathrm{y}}}{2 a\left(E I / L^{3}\right)+P e / L} \\
& =\frac{F_{\mathrm{y}}}{24\left(E I / L^{3}\right)+1.2 P / L}
\end{aligned}
$$

Comparing Eq. (2) and Eq. (3), we can observe that

$$
\left\{\begin{array}{l}
1 / \gamma=1.2 \Rightarrow \gamma=0.833 \\
K_{\mathrm{b}} / \gamma^{2}=6(E I / L) \Rightarrow K_{\mathrm{b}}=4.167 E I / L
\end{array}\right.
$$



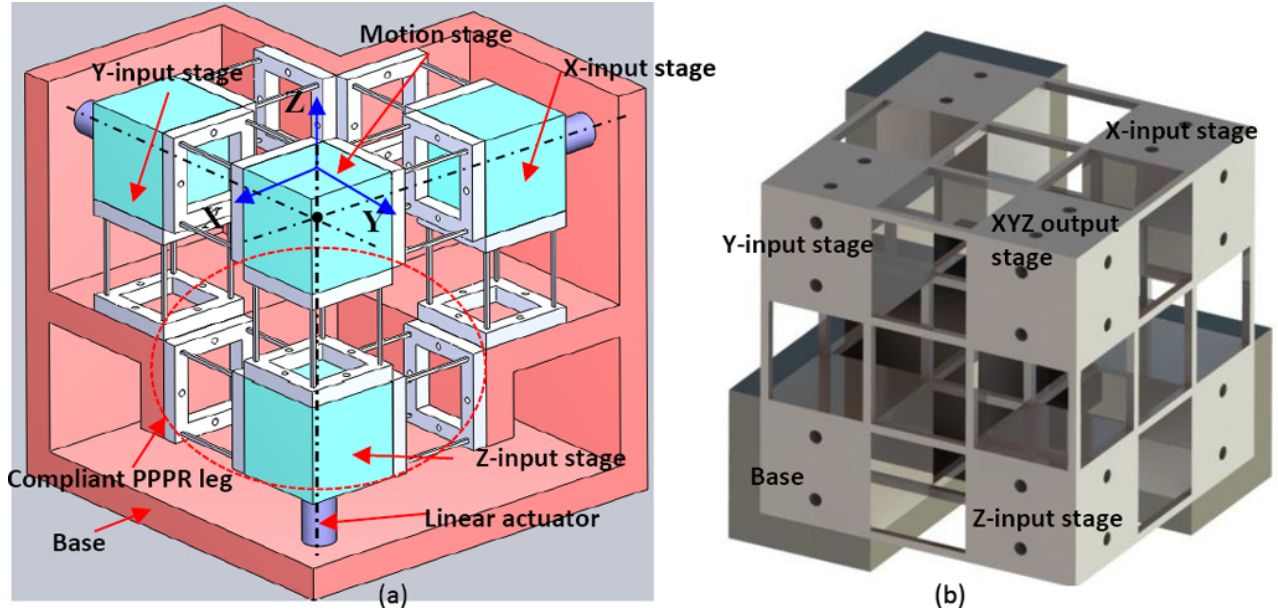

(b)

Figure 2. A compact and decoupled XYZ compliant parallel manipulator composed of identical spatial four-beam modules: (a) 3-PPPR XYZ CPM, and (b) corresponding monolithic design to be fabricated from a cubic material by three orthogonal directions' cutting.

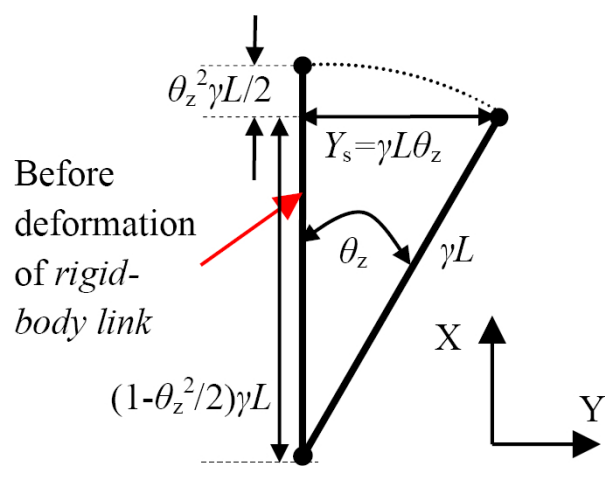

Figure 3. Kinematics schematic diagram of bending about $z$ axis of a fixed-fixed beam.

It is noted that, during the above derivation, the characteristic factor, $\gamma$, is independent of the cross-section shape or the second moment of cross-section area, $I$. From Eq. (4), it can be observed the bending stiffness coefficient $K_{\theta}=2.50$.

\subsection{Simplified PRBMs for spatial multi-beam modules}

The spatial deformation/motion of a fixed-fixed beam within a spatial three-beam module (Fig. 4) can be stimulated via the superposition principle using the results from the independent two planar bending motions (Sect. 2.1) along with the twisting motion. The spatial-motion beam can be accordingly equivalent to a rigid-body link with two identical spherical joints (Wang et al., 2008; Ramirez and Lusk, 2011). Similarly, each spherical joint is located each located the same distance from their respective end and the rigid-body link has a length of $\gamma L(\gamma=0.833)$. For each spherical joint, the twisting stiffness $K_{\mathrm{t}}$ can be simply derived as $K_{\mathrm{t}}=2 G I_{\mathrm{p}} / L=$ $4 G I / L\left(I_{\mathrm{p}}=2 I\right)$, and the bending stiffness $K_{\mathrm{b}}$ about any bend- ing axis is obtained as $K_{\mathrm{b}}=2 \gamma K_{\theta} E I / L=4.167 E I / L$ based on the result derived in Sect. 2.1 (Eq. 4).

According to the virtual work principle and under the assumption of negligible bending rotation (which implies the twisting rotation is very small), we obtain the following expression for the spatial three-beam module (Fig. 4):

$$
\begin{gathered}
F_{\mathrm{y}} \mathrm{d} Y_{\mathrm{s}}+F_{\mathrm{z}} \mathrm{d} Z_{\mathrm{s}}+P \mathrm{~d} X_{\mathrm{s}}+M_{\mathrm{x}} \mathrm{d} \theta_{\mathrm{sx}} \\
=\frac{\partial U}{\partial Y_{\mathrm{s}}} \mathrm{d} Y_{\mathrm{s}}+\frac{\partial U}{\partial Z_{\mathrm{s}}} \mathrm{d} Z_{\mathrm{s}}+\frac{\partial U}{\partial \theta_{\mathrm{sx}}} \mathrm{d} \theta_{\mathrm{sx}}
\end{gathered}
$$

where the variables $Y_{\mathrm{s}}, Z_{\mathrm{s}}$ and $\theta_{\mathrm{sx}}$ are the generalized coordinates along the $y, z$, and $x$ axes, respectively, which are the primary motion displacements of the motion stage center, O', with regard to the fixed coordinate system O-XYZ. $X_{\mathrm{s}}$ is the parasitic translation along the $x$ axis. $F_{\mathrm{y}}, F_{\mathrm{z}}$ and $P$ are the two transverse forces and the axial force along the $y, z$, and $x$ axes, respectively, and $M_{\mathrm{x}}$ is the twisting moment about the $x$ axis. $U$ is the total elastic energy.

As shown in Fig. 4, there are six spherical joints for the spatial three-beam module in its PRBM embodiment. Based on the small range of motion assumption and superposition principle, we have

$$
\begin{aligned}
U & =6 \times \frac{1}{2} K_{\mathrm{b}}\left(\frac{Y_{\mathrm{s}}}{\gamma L}\right)^{2}+6 \times \frac{1}{2} K_{\mathrm{b}}\left(\frac{Z_{\mathrm{s}}}{\left(1-\left(Y_{\mathrm{s}} / \gamma L\right)^{2} / 2\right) \gamma L}\right)^{2} \\
& +6 \times \frac{1}{2} K_{\mathrm{t}}\left(\frac{\theta_{\mathrm{sx}}}{2}\right)^{2}+6 \times \frac{1}{2} K_{\mathrm{b}}\left(\frac{R_{3} \theta_{\mathrm{sx}}}{\gamma L}\right)^{2} \\
X_{\mathrm{s}} & =-\left[1-\cos \left(\frac{Y_{\mathrm{s}}}{\gamma L}\right)\right] \gamma L-\left[1-\cos \left(\frac{Z_{\mathrm{s}}}{\gamma L}\right)\right] \gamma L \\
& -\left[1-\cos \left(\frac{R_{3} \theta_{\mathrm{sx}}}{\gamma L}\right)\right] \gamma L=-\left(\frac{Y_{\mathrm{s}}}{\gamma L}\right)^{2} \gamma L / 2 \\
& -\left(\frac{Z_{\mathrm{s}}}{\gamma L}\right)^{2} \gamma L / 2-\left(\frac{R_{3} \theta_{\mathrm{sx}}}{\gamma L}\right)^{2} \gamma L / 2
\end{aligned}
$$




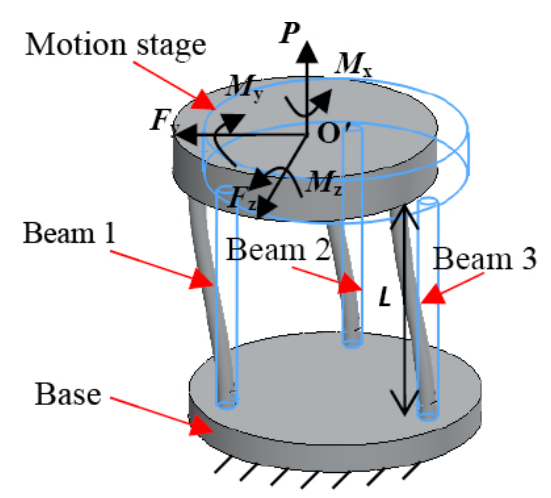

a) Spatial three-beam module in deformation

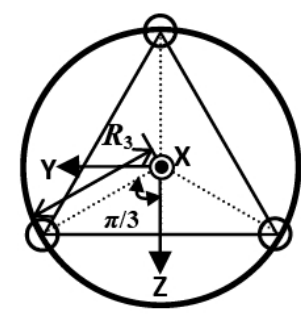

b) Top view of motion stage

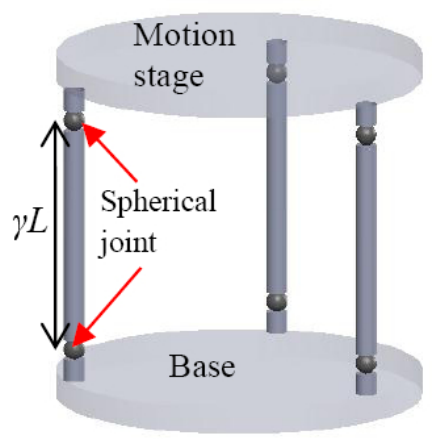

c) Equivalent PRBM embodiment

Figure 4. Spatial three-beam module and the corresponding PRBM.

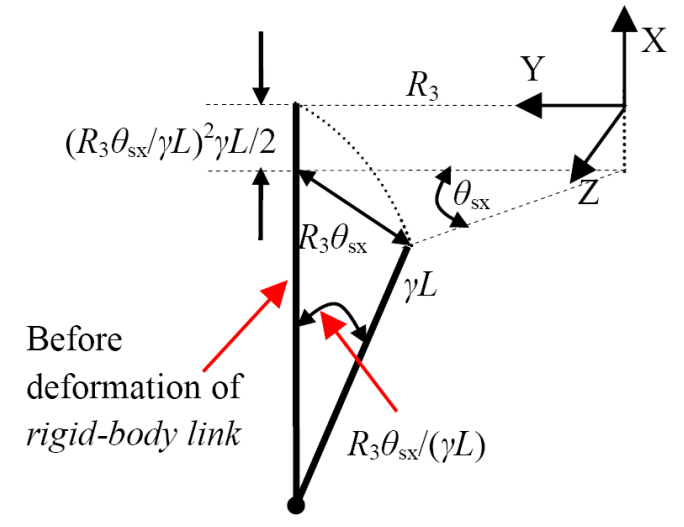

Figure 5. Kinematic schematic diagram of twisting rotation about the $x$ axis of the spatial three-beam module.

where the kinematic relationships used for bending and twisting are shown in Figs. 3 and 5. Moreover, the bending in the XOY plane is assumed to be the first rotation about the $z$ axis, and that in the XOZ plane is assumed to be the second rotation about the $y$ axis. Therefore, $\frac{Y_{\mathrm{s}}}{\gamma L}$ and $\frac{Z_{\mathrm{s}}}{\left(1-\left(Y_{\mathrm{s}} / \gamma L\right)^{2} / 2\right) \gamma L}$ are used herein to denote the rotational angles about the $y$ and $z$ axes, respectively, in order to capture the tiny cross-axis coupling in the two primary translational directions.

Substituting Eqs. (6) and (7) into Eq. (5), we obtain

$$
\begin{gathered}
F_{\mathrm{y}}-P \mathrm{~d}\left[\left(\frac{Y_{\mathrm{s}}}{\gamma L}\right)^{2} \gamma L / 2\right] / \mathrm{d} Y_{\mathrm{s}}=\frac{\mathrm{d}\left[3 K_{\mathrm{b}}\left(\frac{Y_{\mathrm{s}}}{\gamma L}\right)^{2}\right]}{\mathrm{d} Y_{\mathrm{s}}} \\
+\frac{\mathrm{d}\left[3 K_{\mathrm{b}}\left(\frac{Z_{\mathrm{s}}}{\left(1-\left(Y_{\mathrm{s}} / \gamma L\right)^{2} / 2\right) \gamma L}\right)^{2}\right]}{\mathrm{d} Y_{\mathrm{s}}}=\frac{6 K_{\mathrm{b}} Y_{\mathrm{s}}}{(\gamma L)^{2}} \\
+\frac{6 K_{\mathrm{b}} Y_{\mathrm{s}}}{(\gamma L)^{2}} \frac{Z_{\mathrm{s}}^{2} /(\gamma L)^{2}}{\left[1-\left(Y_{\mathrm{s}} / \gamma L\right)^{2} / 2\right]^{3}} \approx \frac{6 K_{\mathrm{b}} Y_{\mathrm{s}}}{(\gamma L)^{2}}+\frac{6 K_{\mathrm{b}} Y_{\mathrm{s}}}{(\gamma L)^{2}} Z_{\mathrm{s}}^{2} /(\gamma L)^{2}
\end{gathered}
$$

$$
\begin{aligned}
= & \frac{6 K_{\mathrm{b}} Y_{\mathrm{s}}}{(\gamma L)^{2}}\left[1+Z_{\mathrm{s}}^{2} /(\gamma L)^{2}\right] \\
\Rightarrow & Y_{\mathrm{s}} \approx \frac{F_{\mathrm{y}}}{6 K_{\mathrm{b}}\left[1+Z_{\mathrm{s}}^{2} /(\gamma L)^{2}\right] /(\gamma L)^{2}+P /(\gamma L)} \\
= & \frac{F_{\mathrm{y}}}{6 K_{\mathrm{b}}\left[1+\left(\frac{F_{\mathrm{z}}}{6 K_{\mathrm{b}} /(\gamma L)^{2}+P /(\gamma L)}\right)^{2} /(\gamma L)^{2}\right] /(\gamma L)^{2}+P /(\gamma L)} \\
F_{\mathrm{z}} & -P \mathrm{~d}\left[\left(\frac{Z_{\mathrm{s}}}{\gamma L}\right)^{2} \gamma L / 2\right] / \mathrm{d} Z_{\mathrm{s}}=\frac{1}{\mathrm{~d}\left[3 K_{\mathrm{b}}\left(\frac{Z_{\mathrm{s}}}{\left(1-\left(Y_{\mathrm{s}} / \gamma L\right)^{2} / 2\right) \gamma L}\right)^{2}\right]} \\
= & \frac{6 K_{\mathrm{b}} Z_{\mathrm{s}}}{(\gamma L)^{2}} \frac{\mathrm{d} Z_{\mathrm{s}}}{\left[1-\left(Y_{\mathrm{s}} / \gamma L\right)^{2} / 2\right]^{2}} \approx \frac{6 K_{\mathrm{b}} Z_{\mathrm{s}}}{(\gamma L)^{2}} \frac{1-\left(Y_{\mathrm{s}} / \gamma L\right)^{2}}{1-\left(\gamma \theta_{\mathrm{sx}}\right.} \\
= & \frac{6 K_{\mathrm{b}} Z_{\mathrm{s}}}{(\gamma L)^{2}} \frac{F_{\mathrm{z}}}{1-\left(Y_{\mathrm{s}} / \gamma L\right)^{4}} \approx \frac{6 K_{\mathrm{b}} Z_{\mathrm{s}}}{(\gamma L)^{2}}\left[1+Y_{\mathrm{s}}^{2} /(\gamma L)^{2}\right] \\
Z_{\mathrm{s}}= & \frac{\mathrm{d}\left[3 K_{\mathrm{t}} \theta_{\mathrm{sx}}^{2} / 4+3 K_{\mathrm{b}}\left(\frac{R_{3} \theta_{\mathrm{sx}}}{\gamma L}\right)^{2}\right]}{6 K_{\mathrm{b}}\left[1+Y_{\mathrm{s}}^{2} /(\gamma L)^{2}\right] /(\gamma L)^{2}+P /(\gamma L)} \\
= & \frac{F_{\mathrm{y}}}{6 K_{\mathrm{b}}\left[1+\left(\frac{F_{\mathrm{y}}}{6 K_{\mathrm{b}} /(\gamma L)^{2}+P /(\gamma L)}\right)^{2} /(\gamma L)^{2}\right] /(\gamma L)^{2}+P /(\gamma L)} \\
M_{\mathrm{x}} & -P \mathrm{~d}\left[\left(\frac{R_{3} \theta_{\mathrm{sx}}}{\gamma L}\right)^{2} \gamma L / 2\right] / \mathrm{d} \theta_{\mathrm{sx}}
\end{aligned}
$$

The dominant kinematic effect component of the bending angle (about the $y / z$ axis) can be then derived using the following purely kinematic relationships between the motion stage center and the tip (mobile end) of the $i$-th beam ( $i=1,2$ and 3) (Hao et al., 2011): 
$X_{1}=X_{\mathrm{s}}-\sqrt{3} R_{3} \theta_{\mathrm{sz}} / 2+R_{3} \theta_{\mathrm{sy}} / 2$

$X_{2}=X_{\mathrm{s}}-R_{3} \theta_{\mathrm{sy}}$

$X_{3}=X_{\mathrm{s}}+\sqrt{3} R_{3} \theta_{\mathrm{sz}} / 2+R_{3} \theta_{\mathrm{sy}} / 2$

$Y_{1}=Y_{\mathrm{s}}-R_{3} \theta_{\mathrm{sx}} / 2$

$Y_{2}=Y_{\mathrm{s}}+R_{3} \theta_{\mathrm{sx}}$

$Y_{3}=Y_{\mathrm{s}}-R_{3} \theta_{\mathrm{sx}} / 2$

$Z_{1}=Z_{\mathrm{s}}+\sqrt{3} R_{3} \theta_{\mathrm{sx}} / 2$

$Z_{2}=Z_{\mathrm{s}}$

$Z_{3}=Z_{\mathrm{s}}-\sqrt{3} R_{3} \theta_{\mathrm{sx}} / 2$

where $X_{i}, Y_{i}$ and $Z_{i}$ are the translational displacements for the $i$-th beam tip along the $x, y$, and $z$ axes.

Based on Figs. 3 and 5, we have the translational displacement along the $x$ axis for each beam tip:

$X_{i}=-\left(\frac{Y_{i}}{\gamma L}\right)^{2} \gamma L / 2-\left(\frac{Z_{i}}{\gamma L}\right)^{2} \gamma L / 2=-\frac{Y_{i}^{2}}{2 \gamma L}-\frac{Z_{i}^{2}}{2 \gamma L}$

Using Eqs. (11), (12) and (13), we can obtain the two bending rotaions about the $y$ and $z$ axes with eliminating the motion stage center displacement, $X_{\mathrm{s}}$, along the $x$ axis as:

$\theta_{\text {sy }}=\frac{\left(X_{1}+X_{3}\right)-2 X_{2}}{3 R_{3}}$

$\theta_{\mathrm{sz}}=\frac{X_{3}-X_{1}}{\sqrt{3} R_{3}}$

Substituting Eq. (20) into Eq. (21), and then substituting Eqs. (14)-(19) to the result, we have

$\theta_{\mathrm{sy}}=\theta_{\mathrm{sx}} Y_{\mathrm{s}} /(\gamma L)$

Similarly, substituting Eq. (20) into Eq. (22), and then substituting Eqs. (14), (16), (17) and (19) to the result, we have

$\theta_{\mathrm{sz}}=\theta_{\mathrm{sx}} Z_{\mathrm{s}} /(\gamma L)$

Analogously, the PRBMs of a class of spatial multi-beam modules that all the beams thereof are uniformly spaced around a circle with a radius of $R_{n}$ ( $n>=3$ and is even for $n \neq 3$ ) can be derived as following:

$$
\begin{aligned}
& Y_{\mathrm{s}}=\frac{F_{\mathrm{y}}}{2 n K_{\mathrm{b}}\left[1+Z_{\mathrm{s}}^{2} /(\gamma L)^{2}\right] /(\gamma L)^{2}+P /(\gamma L)} \\
& Z_{\mathrm{s}}=\frac{F_{\mathrm{z}}}{2 n K_{\mathrm{b}}\left[1+Y_{\mathrm{s}}^{2} /(\gamma L)^{2}\right] /(\gamma L)^{2}+P /(\gamma L)} \\
& \theta_{\mathrm{sx}}=\frac{M_{\mathrm{x}}}{n K_{\mathrm{t}} / 2+2 n K_{\mathrm{b}} R_{n}^{2} /(\gamma L)^{2}+P R_{n}^{2} /(\gamma L)}
\end{aligned}
$$

$$
\begin{aligned}
& X_{\mathrm{s}}=-\left(\frac{Y_{\mathrm{s}}}{\gamma L}\right)^{2} \gamma L / 2-\left(\frac{Z_{\mathrm{s}}}{\gamma L}\right)^{2} \gamma L / 2-\left(\frac{R_{n} \theta_{\mathrm{sx}}}{\gamma L}\right)^{2} \gamma L / 2 \\
& \theta_{\mathrm{sy}}=\theta_{\mathrm{sx}} Y_{\mathrm{s}} /(\gamma L) \\
& \theta_{\mathrm{sz}}=\theta_{\mathrm{sx}} Z_{\mathrm{s}} /(\gamma L)
\end{aligned}
$$

where the loading and displacements are defined in a similar way as mentioned above. The axial force in the denominator terms of Eqs. (25), (26) and (27) causes the load-stiffening effect. When the primary translation stiffness and the twisting stiffness are zero, two values of the axial force $P$ are obtained. The minimal absolute value of the axis force is the buckling load, which is equal to $10 n E I / L^{2}$. In addition, the cross-axis coupling is captured for the two primary translational directions.

Note that Eq. (27) can still be used to estimate the large twisting rotation under the action of the dominant twisting moment only although the twisting rotation is assumed to be very small during the above derivation.

When translational displacements and length parameters are normalized by the beam length $L$, forces by $E I / L^{2}$, moments by $E I / L$, and all normalized results are denoted by their lower-case letters, Eqs. (25)-(30) can be re-written as

$$
\left\{\begin{array}{l}
y_{\mathrm{s}} \approx \frac{f_{\mathrm{y}}}{12 n\left(1+1.44 z_{\mathrm{s}}^{2}\right)+1.2 p} \\
z_{\mathrm{s}} \approx \frac{f_{\mathrm{z}}}{12 n\left(1+1.44 y_{\mathrm{s}}^{2}\right)+1.2 p} \\
\theta_{\mathrm{sx}} \approx \frac{m_{\mathrm{x}}}{n\left(2 G / E+12 r_{n}^{2}+1.2 p r_{n}^{2} / n\right)} \\
x_{\mathrm{s}} \approx-0.6\left(y_{\mathrm{s}}^{2}+z_{\mathrm{s}}^{2}+r_{n}^{2} \theta_{\mathrm{sx}}^{2}\right) \\
\theta_{\mathrm{sy}}=1.2 \theta_{\mathrm{sx}} y_{\mathrm{s}} \\
\theta_{\mathrm{sz}}=1.2 \theta_{\mathrm{sx}} z_{\mathrm{s}}
\end{array}\right.
$$

Comparing Eq. (30) with the previously reported analytical work (Hao et al., 2011), we can see that the present PRBMs are well coincident with the dominant terms of the associated nonlinear analytical results.

For a spatial multi-beam module as applied in Figs. 1 and 2 , its twisting rotation can be negligible. Equations (25), (26) and (28) are therefore capable of determining the three translational displacements for a general spatial module with total $n$ beams no matter how these beams are distributed.

\section{FEA result comparisons}

In order to verify the accuracy of the present PRBMs of spatial multi-beams, an example spatial three-beam module (Fig. 4) is analyzed using nonlinear FEA software (Comsol). The spatial three-beam module is taken to be made from a standard aluminium alloy for which Young's modulus, $E$, is $69000 \mathrm{~N} \mathrm{~mm}^{-2}$ and Poisson's ratio, $v$, is 0.33 . The beam has round cross-section with a diameter of $D=4 \mathrm{~mm}$. The other geometrical parameters are set as $R_{3}=30 \mathrm{~mm}$ and $L=50 \mathrm{~mm}$. 


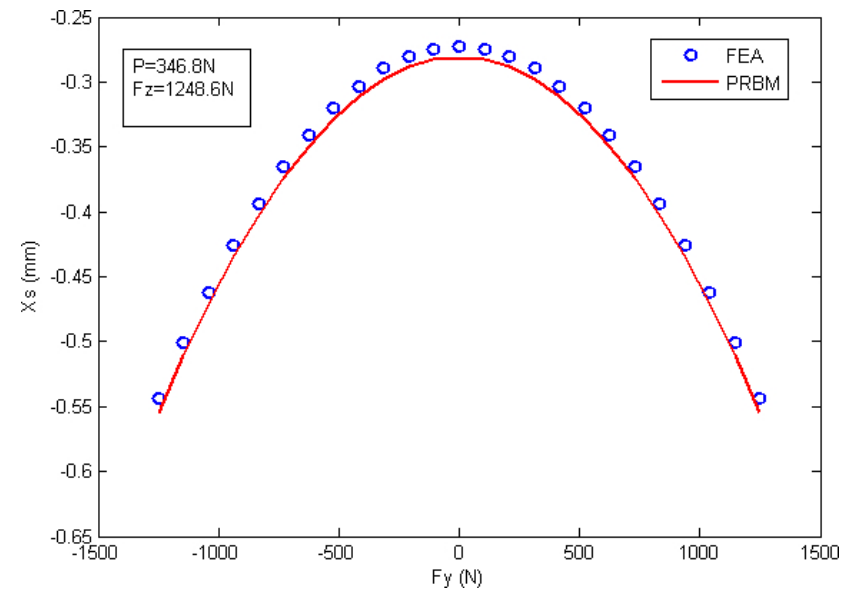

Figure 6. Parasitic translational displacement verification.

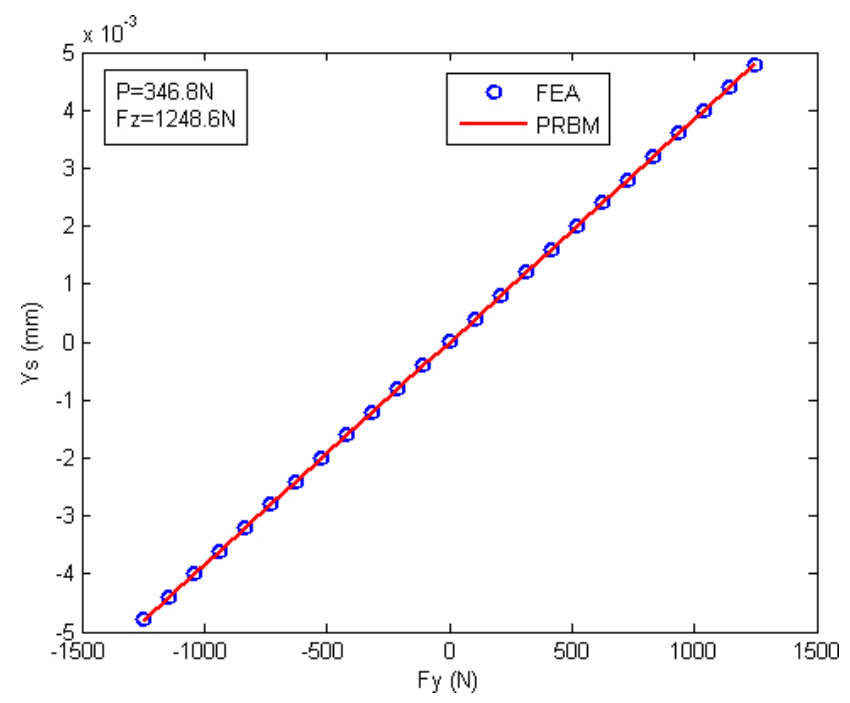

Figure 7. Primary translational displacement verification: primary stiffness.

FEA results with comparisons with the PRBM results for the spatial three-beam module are shown in Figs. 6-11. It is shown in Figs. 6, 7, 8 that the translational displacements, including the primary motion and parasitic motion, obtained from the FEA have a good agreement with those obtained from the PRBM. The maximal differences in percentage (FEA results as the denominator) in Figs. 6, 7, 8 are $2.18 \%, 0.40 \%$ and $0.43 \%$, respectively.

The FEA results and the PRBM results both capture the cross-axis coupling effect in the two primary translational directions with an acceptable difference (Fig. 9), which describes that the cross-axis force slightly increases the primary translational stiffness. The maximal cross-axis coupling error from the FEA is of $0.83 \%$, and that from the PRBM is $1.24 \%$, which suggests that the cross-axis coupling in two primary translational directions can be ignored.

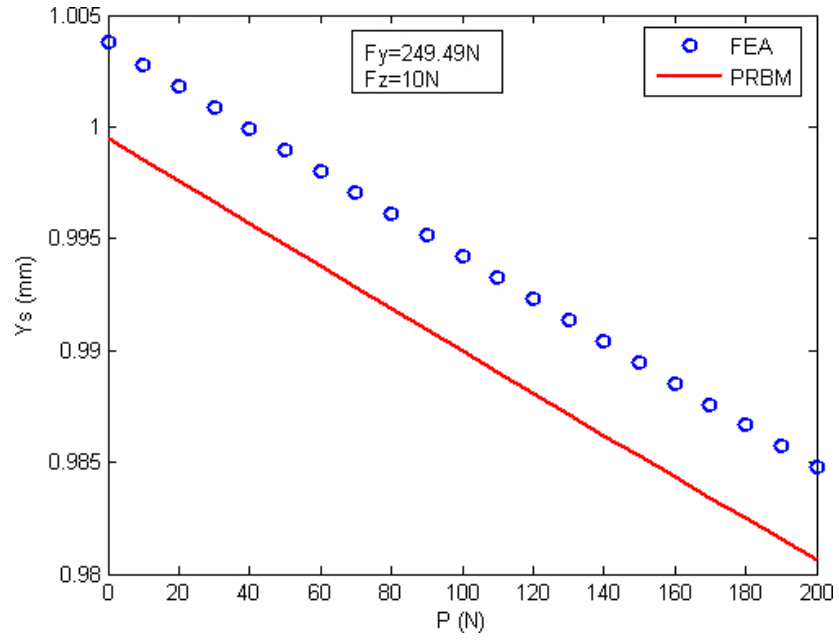

Figure 8. Primary translational displacement verification: crossaxis coupling effect caused by the axial force.

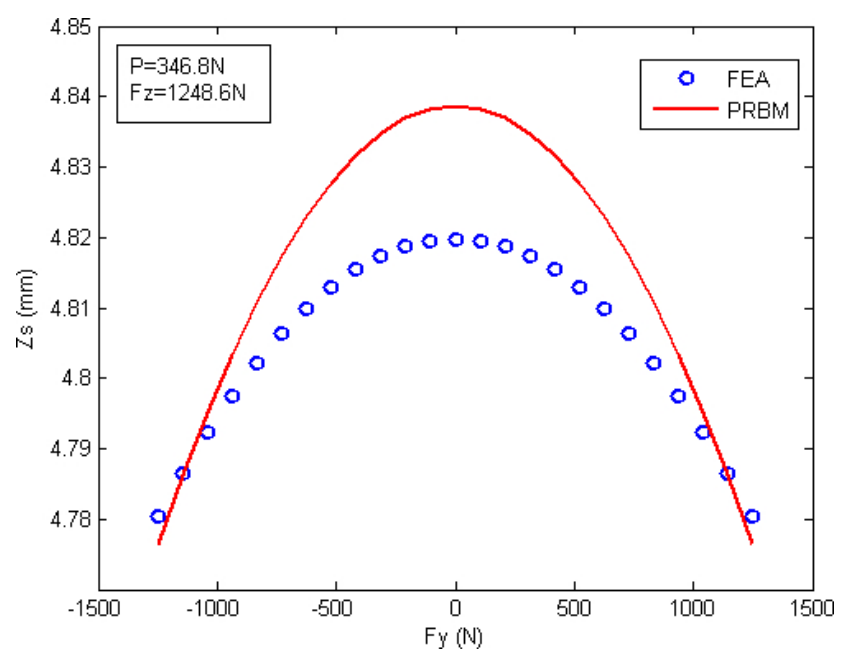

Figure 9. Primary translational displacement verification: crossaxis coupling effect in the two primary translational directions.

Figure 10 shows that the twisting angle about the $\mathrm{X}$-axis obtained from the FEA results is within $10 \mu \mathrm{rad}$ (most probably from the inaccuracy of the FEA results) compared with the zero value obtained from the PRBM results when only pure forces are exerted on the motion stage center.

In addition, the FEA results capture the bending rotation, $\theta_{\mathrm{sz}}$, under the dominant force, $F_{\mathrm{y}}$, with the value less than $1.5 \mathrm{mrad}$ (Fig. 11), which is not obtained by the PRBM results (Eq. 30). From the analytical results in Hao et al. (2011), the bending rotation effect can be smaller when $R_{3}$ increases and/or $D$ decreases. The dominant kinematic effect component of the bending angle $\left(\theta_{\mathrm{sz}}\right)$ caused by the non-dominant coupled loads $\left(M_{\mathrm{x}}\right.$ and $F_{\mathrm{z}}$ ) (Eq. 30$)$ has been roughly verified by a prototyped spatial three-beam module in Hao et al. (2011). 


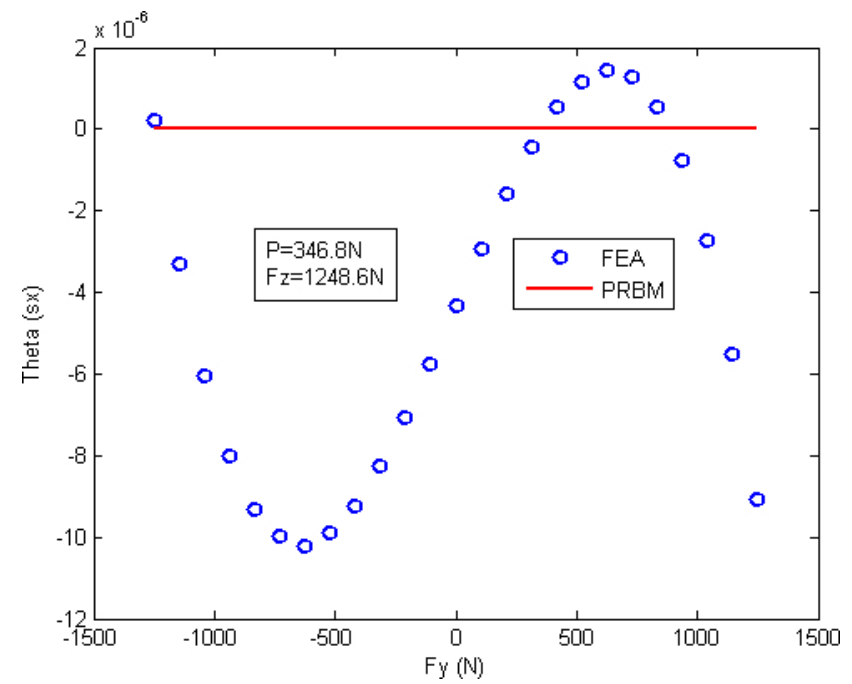

Figure 10. Twisting angle about the $x$ axis.

\section{Discussions}

As shown in PRBMs proposed in this paper and/or Figs. 6 and 11, the purely elastic effect of the axis force along the $x$ axis and the parasitic bending rotation caused by the dominant force (or moment) are lost, which is the main shortcoming of the PRBMs. One alternative approach to overcome this issue is to use the PRBMs for determining the two primary translational motions and to use the analytical results in Hao et al. (2011) for capturing the other characteristics. The PRBMs of spatial multi-beam modules may be re-derived via using three spherical joints in each leg similar to the planar motion case reported in $\mathrm{Su}$ (2009).

In addition, the PRBM for a fixed-fixed beam in spatial motion can be modified via the use of the recently developed results to capture the coupling between the two bending directions in the presence of a torsional load (Sen and Awtar, 2013), and/or the kinematic and elastokinematic components of twisting angle in the presence of bending displacements (Hao et al., 2011; Sen and Awtar, 2013).

\section{Conclusions}

Simplified PRBMs have been presented in this paper to deal with the spatial multi-beam modules with planar motion for specific applications/loading conditions via replacing each beam with a rigid-body link plus two identical spherical joints. The characteristics factor, bending stiffness and twisting stiffness for the spherical joint have been determined. These presented load-displacement equations have been verified by nonlinear FEA software, which can be used to predict the nonlinear characteristics such as load-stiffening effect, cross-axis coupling in the two primary translational directions and buckling load.

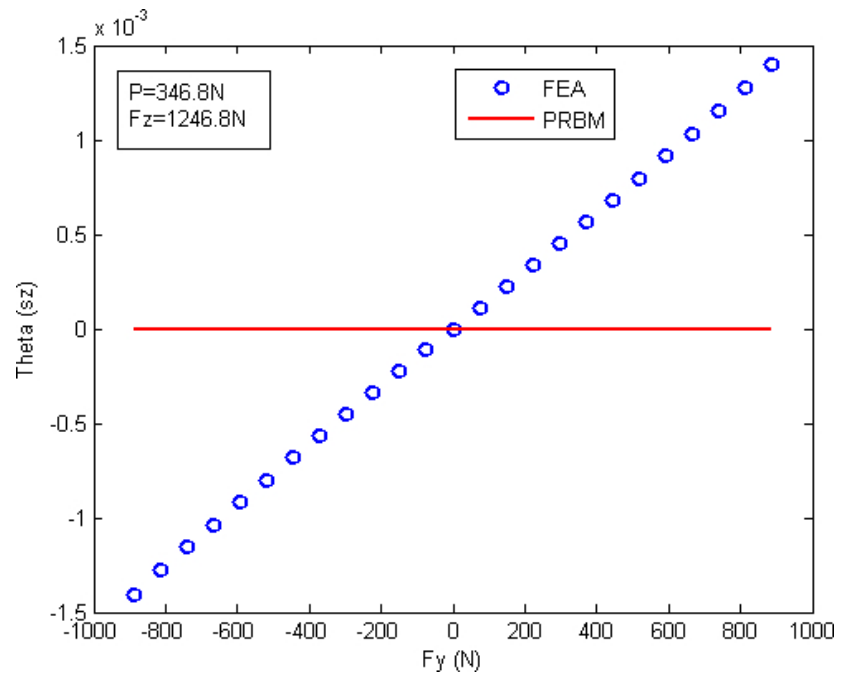

Figure 11. Bending angle about the $z$ axis.

It is noted that the PRBM proposed in this paper can be further improved to capture the additional nonlinear characteristics such as the purely elastic effect of axis force along the $x$ axis, and the bending rotation caused by the dominant load. The PRBMs for more generality with diverse loading conditions also deserve the future investigations.

Acknowledgements. The author would like to thank final year undergraduates John Mullins and Mark Bruton in UCC for their contribution to Fig. 1.

Edited by: H. Su

Reviewed by: C. Lusk and one anonymous referee

\section{References}

Awtar, S. and Slocum, A. H.: Characteristics of Beam-Based Flexure Modules, J. Mech. Design, 129, 624-639, 2007.

Culpepper, M., DiBiasio, C., Panas, R., Magleby, S., and Howell, L. L.: Simulation of a Carbon Nanotube-Based Compliant ParallelGuiding Mechanism: A Nanomechanical Building Block, Appl. Phys. Lett., 89, 203111, doi:10.1063/1.2388143, 2006.

Hao, G. and Kong, X.: Novel XY Compliant Parallel Manipulators for Large Translation with Enhanced Out-of-Plane Stiffness, J. Mech. Design, 134, 061009, doi:10.1115/1.4006653, 2012a.

Hao, G. and Kong, X.: Design and Modelling of a Large-Range Modular XYZ Compliant Parallel Manipulators Using Identical Spatial Modules, Journal of Mechanisms and Robotics, 4, 021009, doi:10.1115/1.4006188, 2012b.

Hao, G., Kong, X., and Reuben, R. L.: A Nonlinear Analysis of Spatial Compliant Parallel Modules: Multi-beam Modules, Mech. Mach. Theory, 46, 680-706, 2011.

Howell, L. L.: Compliant Mechanisms, Wiley, New York, 2001.

Howell, L. L., Midha A., and Norton, T. W.: Evaluation of Equivalent Spring Stiffness for Use in a Pseudo-Rigid-Body Model of 
Large-Deflection Compliant Mechanisms, J. Mech. Design, 118, 126-131, 1996.

Howell, L. L., DiBiasio, C. M., Cullinan, M. A., Panas, R., and Culpepper, M. L: A Pseudo-Rigid-Body Model for Large Deflections of Fixed-Clamped Carbon Nanotubes, Journal of Mechanisms and Robotics, 2, 034501-1-034501-5, 2010.

Ramirez, I. A. and Lusk, C. P.: Spatial-Beam Large-Deflection Equations and Pseudo-Rigid Body Model for Axisymmetric Cantilever Beams, Proceedings of the ASME 2011 International Design Engineering Technical Conferences \& Computers and Information in Engineering Conference, IDETC/CIE 2011, 29-31 August 2011, Washington, DC, USA, DETC2011-47389, 2011.
Sen, S. and Awtar, S.: A Closed-Form Nonlinear Model for the Constraint Characteristics of Symmetric Spatial Beams, J. Mech. Design, 135, 031003, doi:10.1115/1.4023157, 2013.

Su, H.-J.: A Pseudo-Rigid-Body 3R Model for Determining Large Deflection of Cantilever Beams Subject to Tip Loads, Journal of Mechanisms and Robotics, 1, 021008, doi:10.1115/1.3046148, 2009.

Wang, Z., Pan, Y., Ji, S., Su, J., and Wan, Y.: A Family of Spatial Translational Parallel Robots, Proceedings of 7th World Congress on Intelligent Control and Automation, 25-27 June 2008, Chongqing, China, 2008. 